\title{
Cyclic variability of stromal cell-derived factor-1 and endothelial progenitor cells during the menstrual cycle
}

\author{
ELZAFIR ELSHEIKH ${ }^{1}$, CHRISTER SYLVÉN $^{2}$, BO-GÖRAN ERICZON ${ }^{1}$, JAN PALMBLAD $^{3}$ and MIRIAM MINTS ${ }^{4}$ \\ ${ }^{1}$ Division of Transplantation Surgery, Department of Clinical Science, Intervention and Technology, \\ Karolinska Institutet at Karolinska University Hospital Huddinge, S-14186 Stockholm; ${ }^{2}$ Department of Medicine, \\ Division of Cardiology, Karolinska Institutet at Karolinska University Hospital Huddinge, Stockholm; \\ ${ }^{3}$ Center for Inflammation and Hematology Research, Department of Medicine, Karolinska Institutet \\ at Karolinska University Hospital Huddinge, Stockholm; ${ }^{4}$ Department of Women's and Children's Health, \\ Karolinska Institutet, Karolinska University Hospital, Solna, S-17176 Stockholm, Sweden
}

Received August 17, 2010; Accepted October 12, 2010

DOI: $10.3892 / \mathrm{ijmm} .2010 .570$

\begin{abstract}
The endometrium goes through a unique cycle of physiological angiogenesis during the normal menstrual cycle (MC). We studied whether there is a correlation between endothelial progenitor cells (EPCs) and plasma and endometrial levels of angiogenic growth factors during the MC. Ten healthy, regularly menstruating women provided blood samples and another 16 supplied endometrial biopsies. Blood samples were obtained over a single MC: twice in the proliferative and once in the secretory phase and at ovulation. Endometrial biopsies were provided in the proliferative or in the secretory phase. We assessed plasma levels of vascular endothelial and fibroblast growth factors, granulocyte and granulocyte-macrophage colony-stimulating factors and stromal cell-derived factor-1 (SDF-1) by ELISA; EPCs by a colony-forming unit (CFU) assay; immunostaining for endometrial SDF-1 by computer-assisted software; and endothelial cell (EC) markers by flow cytometry. In the proliferative phase, SDF-1 levels were significantly higher than during the secretory phase. EPC-CFUs correlated negatively to SDF-1 levels. Endometrial SDF-1 expression tended to be higher in the secretory than in the proliferative phase. Furthermore, vascular endothelial growth factor receptors and Tie-2 EPCs showed a cyclic pattern over the MC. Our results point to SDF-1 as a novel mediator of EPC trafficking during the MC.
\end{abstract}

Correspondence to: Dr Elzafir Elsheikh, Department of Clinical Science, Intervention and Technology (CLINTEC), Division of Transplantation Surgery F82, Karolinska Institutet at Karolinska University Hospital Huddinge, S-14186 Stockholm, Sweden E-mail: elzafir.elsheikh@ki.se

Key words: stromal cell-derived factor-1, endothelial progenitor cells, menstrual cycle, endometrium

\section{Introduction}

The endometrium is a remarkable example of controlled tissue remodeling, unparalleled in other organs. A prerequisite to menstruation is the breakdown of both blood vessels and of the surface epithelium. Repair of the endometrium begins as early as $36 \mathrm{~h}$ after the onset of menstruation. Within 5-6 days, the old lining is removed and a new one regenerates without scarring (1).

The endometrium has a well-developed vasculature. Angiogenesis is an essential component of the regeneration of the endometrium. Based on animal and in vitro studies, angiogenesis is believed to occur in three ways. The first is by sprouting, migration and tube formation of endothelial cells (ECs) in existing vessels, to form new vessels. The second is by intussusceptions, the insertion of connective tissue strands in the lumen of existing vessels, dividing them into more lumina (2). The third is the import of endothelial progenitor cells (EPCs) from the bone marrow, to cover the surface of new vessels (3). In a previous study, we showed that blood vessels in the endometrium develop from EPCs derived from the bone marrow: we found 14\% donor-derived cells in endometrial blood vessels of a human leukocyte antigen (HLA)-mismatched, bone marrow-transplanted woman (4). Recently, Robb et al confirmed that EPCs are present in the endometrium and that the number of these cells varies during the menstrual cycle (MC) (5).

EPCs are a subtype of bone marrow-derived progenitor cells (6) that can enter the peripheral circulation, differentiate into mature ECs, and participate in endothelial repair (7). Currently, EPCs are phenotypically defined as a triplepositive cell population expressing CD $133^{+} / \mathrm{CD} 34^{+} / \mathrm{VEGFR}^{-} 2^{+}$ (vascular endothelial growth factor receptor-2) surface markers (8). However, the exact role of this population is still debated (9). The in vitro function and clonogenic capacity of these EPCs have been evaluated using colony-forming unit (CFU) assays (10), which are used as a biological marker for vascular diseases. However, these colonies also contain myeloid cells (9), which will not be able to differentiate into a functioning endothelial monolayer. In addition to EPCs in 
the circulation, mature ECs or/and inflammatory ECs (IECs), having detached from the vessel walls, may be found in the blood as a result of vascular injury $(11,12)$.

The aim of the present study was to investigate the variation in the numbers of EPCs and EPC colonies during the MC and their correlation with the blood levels of some angiogenesis growth factors. These include the vascular endothelial growth factor (VEGF $\mathrm{A}_{165}$ ) and the basic fibroblast growth factor (FGF). In addition, we studied the granulocyte and granulocyte macrophage colony-stimulating factors (Gand GM-CSF), which are involved in the release of myeloid progenitor cells from the bone marrow and can accelerate neovascularization $(13,14)$. Finally, we assessed a pivotal factor for release and homing of bone marrow-derived cells, including myeloid progenitors and EPCs, namely the stromal cell-derived factor-1 (SDF-1) $(15,16)$.

\section{Material and methods}

Subjects. Blood samples were collected from 10 normal, healthy, ovulating women (mean age $33 \pm 6$ years). All women had a regular MC of 25-32 days. None of the women had used hormonal or intrauterine contraception for at least 3 months prior to the study.

The MC stage was based on the date of last menstruation and the analysis of estradiol and progesterone. Participants identified the day of the ovulation (luteal hormone surge) by testing their morning urine.

Blood samples were collected in the early proliferative phase (days 1-2), mid-proliferative phase (day 5), at the time of ovulation (day 14) and in the secretory phase (days 22-24). The blood was used for isolation of peripheral blood mononuclear cells (PBMCs) and culturing of EPCs. Ethylenediamine-tetra-acetic acid plasma samples (obtained by centrifugation at $1,000 \mathrm{xg}$ ), collected at the same time points as mentioned above, were frozen at $-70^{\circ} \mathrm{C}$ until analysis.

Endometrial biopsies were obtained from an additional set of 16 healthy women using a thin plastic catheter for endometrium samples (Pipelle Laboratoire CCD, Paris, France). Nine women were in the proliferative phase and seven were in the secretory phase. Details as to the subjects and procedures have been given $(17,18)$.

Approval for these studies was obtained from the Karolinska University Hospital institutional review board. Informed consent was provided in accordance with the Declaration of Helsinki.

EPC colony assay. To study the functional capacity of EPCs during menstrual angiogenesis, we performed the EPC colony assay. A two-step colony-forming assay was performed to detect the number of EPC colonies, as described by Hill et al (10). Briefly, PBMCs, isolated at the different time points mentioned, were cultured on fibronectin $(20 \mu \mathrm{g} / \mathrm{ml})$-coated tissue culture 6 -well plates $\left(5 \times 10^{6}\right.$ cells/well), in endothelialselective medium (EndoCult; Stemcell Technologies Inc., Vancouver, BC, Canada) in an incubator $\left(37^{\circ} \mathrm{C}, 95 \%\right.$ humidity, $5 \% \mathrm{CO}_{2}$ ). On day 2 , the non-adherent cells were collected and plated in another fibronectin-coated plate. The number of colonies formed from the different wells was counted at day 5 after culturing.
Levels of angiogenic growth factors during angiogenesis. Plasma samples were assayed for VEGF, G-CSF, GM-CSF, FGF, and SDF-1 levels by means of an enzyme-linked immunosorbent assay (ELISA; R\&D Systems, Minneapolis, MN, USA), according to the manufacturer's instructions. Recombinant human VEGF, G-CSF, GM-CSF, FGF, or SDF-1 were used as standards. Determinations for each growth factor were made in a single plate and read by a kinetic microplate reader (VMax). The intra-assay CV range was 3-9\%.

Immunohistochemistry. Endometrium sections were stained with a rabbit anti-human SDF-1 antibody $(2 \mu \mathrm{g} / \mathrm{ml}$ Abcam, Cambridge, MA, USA) in blocking buffer for $1 \mathrm{~h}$ at room temperature. Immunolabeling was performed using the Alexa Fluor 488 goat anti-rabbit anitbody $(5 \mu \mathrm{g} / \mathrm{ml}$; Invitrogen, Stockholm, Sweden). The stained cells were embedded in diamidino-2-phenylindole (DAPI) mounting medium and analyzed by fluorescence microscopy. Histopathological examinations were made by an independent pathologist.

Areas with intense SDF-1 staining ('hot spots') were counted at a $\times 40$ magnification on photographs taken by an LCD camera (Olympus, Japan) connected to a microscope. Between one and three photographs/subject of the positive areas were taken and analyzed blindly by an image analysis program according to preset criteria regarding the size and developed color (Micro Imaging, Olympus). For each subject, the mean of two to three positive areas is given in $\mu \mathrm{m}^{2}$.

Flow cytometry. We were interested in finding out which cell types in the circulation may correlate with endometrium formation. For this purpose, PBMCs were phenotyped with an array of antibodies to specific markers expressed in EPCs (VEGFR-2, VEGFR-1, Tie-2, CD133, CD146, and CD34), mature ECs (CD144, CD141 and CD31), as well as IECs [vascular adhesion protein-1 (VAP-1), CD142, heparan sulphate proteoglycan (HSP), and plasminogen activator inhibitor-1 (PAI-1)].

Antibodies were purchased as follows: anti-VEGFR-2 (RELIATech, Mascheroder, Germany); anti-VEGFR-1 (R\&D Systems); anti-CD133 (Miltenyi Biotec, GmbH, Bergisch, Germany); anti-VAP-1 (Serotec, Düsseldorf, Germany); antiCD141, anti-PAI-1 and anti-HSP (Biogenesis Ltd., Poole, UK). All other antibodies (Tie-2, CD146, CD34, CD144, CD31 and CD142) were purchased from Becton-Dickinson (San Jose, CA, USA). Corresponding control isotypes were used for evaluation of non-specific binding of monoclonal antibodies. The cells were analyzed on a Becton-Dickinson fluorescence-activated flow cytometer (FACSorter).

Statistical analysis. The Friedman non-parametric test was used to test for significant differences in repeated estimates. The non-parametric Wilcoxon matched-pairs test was used to compare levels of growth factors and the number of colonies at the different time points. For the statistical correlations between the growth factors and the number of colonies, the Spearman's test was used. Statistical analysis was performed with the SPSS 17 software package (SPSS Inc., Chicago, IL, USA). Results were considered significant at $p<0.05$. Data for number of EPC colonies, and angiogenic growth factors are presented as medians and interquartile ranges (IQR). 
A)

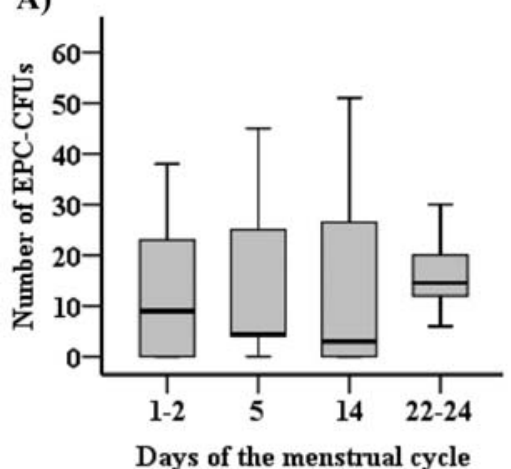

C)

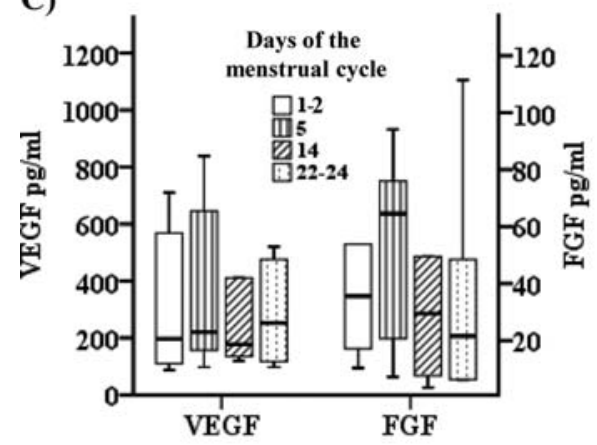

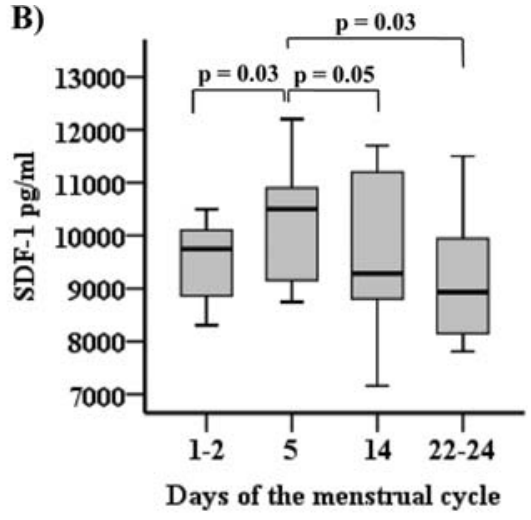

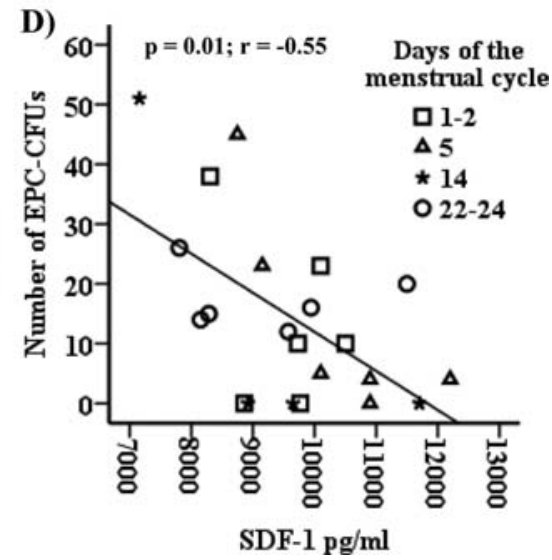

Figure 1. (A) Box plots showing the number of endothelial progenitor cell colony-forming units (EPC-CFUs)/well during the different time points of the menstrual cycle $(\mathrm{MC})(\mathrm{n}=10)$. Values are given as medians and interquartile ranges (IQR); error bars represent minimum and maximum values. (B) Box plots showing stromal cell-derived factor-1 (SDF-1) levels during different time points of the MC. SDF-1 levels increased over the MC, with the levels being highest during the mid-proliferative (day 5: $10,500 \mathrm{pg} / \mathrm{ml}$ ) and lowest during the secretory phase (days 22-24: 8,930 pg/ml; $\mathrm{p}<0.05$ ); however, there was no significant difference between ovulation day and secretory phase $(n=6)$. Values are given as medians and IQR; error bars represent minimum and maximum values. (C) Vascular endothelial growth factor (VEGF) and fibroblast growth factor (FGF) levels during different time points of the MC (n=6). FGF showed a tendency to increase during the MC. Values are given as medians and IQR; error bars represent minimum and maximum values. (D) A scatter plot showing negative correlation between SDF-1 levels (pg/ml, $\mathrm{x}$-axis) and the numbers of circulating EPCs (EPC-CFUs, $\mathrm{y}$-axis); $\mathrm{r}=\mathrm{Spearman}$ 's rank correlation coefficient.

\section{Results}

$E P C-C F U s$. We found that the number of EPC-CFUs showed a large scatter at ovulation, with a median of two CFUs. During the secretory phase, the distribution was more homogeneous, with a tendency of increased numbers of CFUs (15 colonies). However, this difference was not statistically significant (Fig. 1A).

Plasma levels of angiogenic growth factors. Of the angiogenic growth factors tested, the levels of the chemokine SDF-1 significantly varied during the $\mathrm{MC}(\mathrm{p}=0.03)$ (Fig. 1B). SDF-1 plasma levels increased from the early proliferative phase (median, $9749 \mathrm{pg} / \mathrm{ml}$, IQR, 8862-10100 pg/ml), reaching the highest level at the mid-proliferative phase of the MC (median, $10500 \mathrm{pg} / \mathrm{ml}, \mathrm{IQR}, 9151-10900 \mathrm{pg} / \mathrm{ml}$; $\mathrm{p}=0.03$ ), whereafter there was a decrease at ovulation (day 14), reaching the lowest level in the secretory phase (median, 8,930 pg/ml, IQR, 8152$9943 \mathrm{pg} / \mathrm{ml}$ ). The growth factor FGF showed a statistically non-significant pattern $(\mathrm{p}=0.08)$ similar to $\mathrm{SDF}-1$, i.e., a decrease from the mid-proliferative phase (median $64 \mathrm{pg} / \mathrm{ml}$, IQR, 20-75 pg/ml) to the secretory phase (median, $21 \mathrm{pg} / \mathrm{ml}$, IQR, 6-48 pg/ml) of the MC (Fig. 1C).

The levels of systemic VEGF in this study were not significantly different at the different time points studied during the MC (Fig. 1C). In addition, we found no detectable levels of GM-CSF or G-CSF.

Correlation of SDF-1 levels and EPC-CFUs. As shown in Fig. 1D, SDF-1 levels correlated significantly and negatively with EPC-CFU formation ( $\mathrm{r}=-0.55 ; \mathrm{p}=0.01)$. SDF-1 levels were higher during the mid-proliferative phase of the MC than during the secretory phase $(\mathrm{p}=0.03)$, while an opposite tendency was found for EPC-CFUs ( $\mathrm{p}=0.53$ ) (Table I).

Staining of SDF-1 in the endometrial sections. As SDF-1 levels in the circulating blood varied during the MC, we studied the local expression of this chemokine in the endometrium. SDF-1-positive hot spot areas were found in $5 / 9$ and $3 / 7$ of the specimens from the proliferative and secretory phases, respectively. There was a tendency of increased levels of SDF-1 in the secretory phase compared to the proliferative phase $(\mathrm{p}=0.05)$ (Fig. 2A; Table I).

Circulating EPCs and ECs during the MC. The VEGFR-1and VEGFR-2-positive EPCs in the circulation showed a tendency to increase (from $2.03 \%$ to $3.01 \%$; $\mathrm{p}=0.33$ and from $2.49 \%$ to $4.07 \%$; $\mathrm{p}=0.82$, respectively) over the $\mathrm{MC}$, starting from the early proliferative phase, while Tie-2-positive EPCs showed a tendency to decrease (from $0.48 \%$ to $0.08 \%$; 


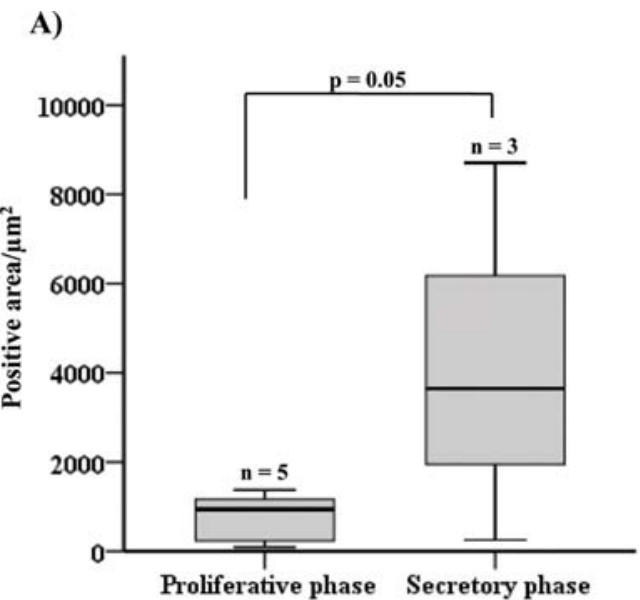

B)

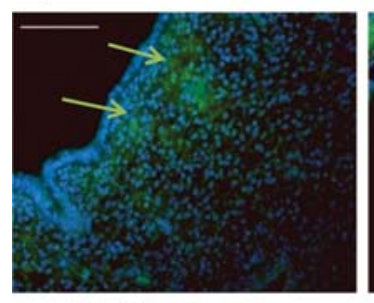

Proliferative phase

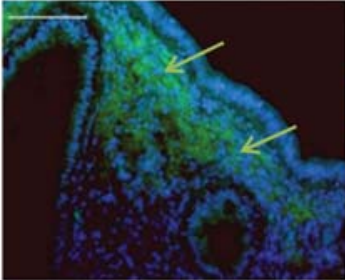

Secretory phase

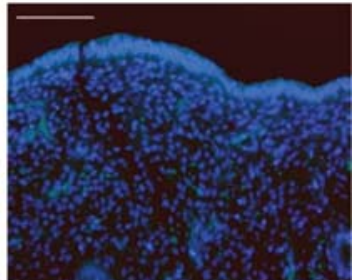

Isotype negative control

Figure 2. (A) The expression of stromal cell-derived factor-1 (SDF-1) in endometrial sections. Levels of this chemokine were higher in the secretory phase than during the proliferative phase $(\mathrm{p}=0.05)$. The level of SDF-1 was quantified using an image analysis program. Values are given as medians and interquartile ranges; error bars represent minimum and maximum values. (B) Representative pictures of the immunohistochemical staining of SDF-1 in the endometrial sections visualized by fluorescent microscopy. Arrows indicate hot spots. Bar, $100 \mu \mathrm{m}$.

A)

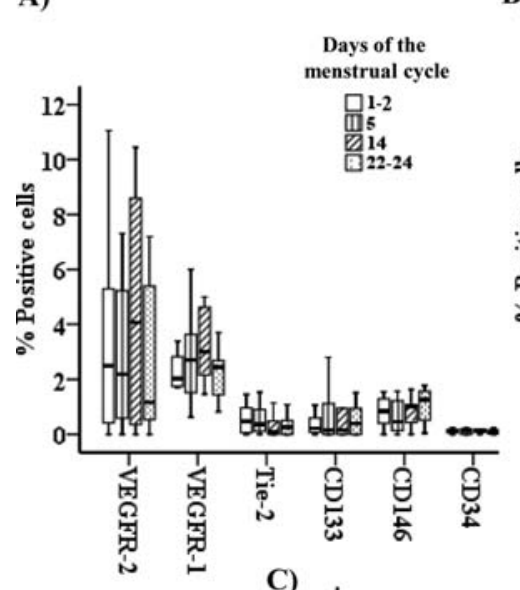

B)

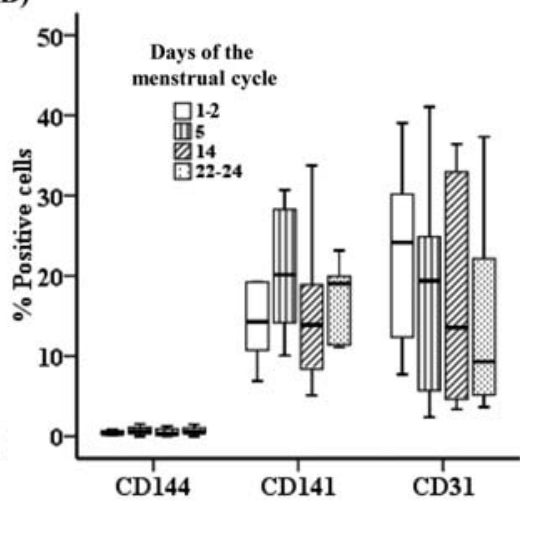

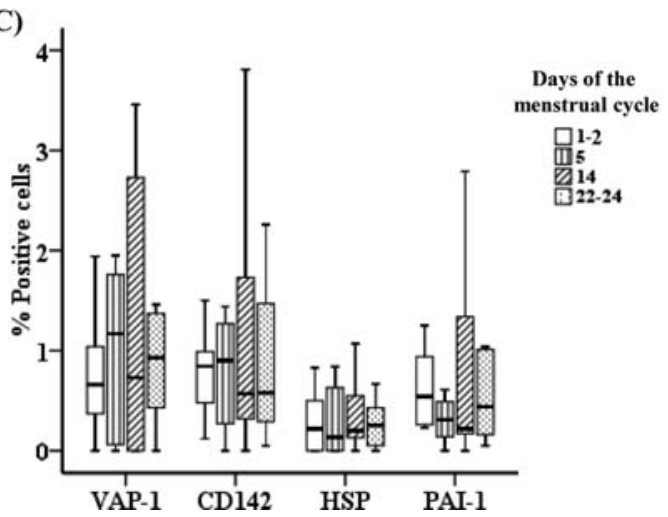

Figure 3. Characterization of endothelial cell (EC) markers in the peripheral blood of normal women during the menstrual cycle (MC). (A) Percentage of cells expressing endothelial progenitor cell (EPC) markers. (B) Percentage of cells expressing mature EC markers. (C) Percentage of inflammatory ECs (IECs). Values are given as medians and interquartile ranges; error bars represent minimum and maximum values; $\mathrm{n}=10$ except for CD34, CD141, PAI-1, and VEGFR-1 ( $\mathrm{n}=6)$. 
$\mathrm{p}=0.67)\left(\right.$ Fig. 3A). We found that the number of $\mathrm{CD} 34^{+}$cells were negatively correlated to the SDF-1 level in the circulation $(\mathrm{r}=-0.549 ; \mathrm{p}=0.01)$. However, the number of $\mathrm{CD} 34^{+}$cells did not vary during the MC (Fig. 3A).

Mature CD31 ECs and inflammatory PAI-1 ECs showed a decreasing pattern during the MC (from $24.16 \%$ to $9.3 \%$; $\mathrm{p}=0.16$ and from $0.54 \%$ to $0.22 \% ; \mathrm{p}=0.78$, respectively) (Fig. 3B and C).

\section{Discussion}

This is, to our knowledge, the first study to show variations in plasma and endometrial concentrations of SDF-1 during the MC. Moreover, we found a highly significant inverse correlation between plasma SDF-1 levels and EPC-CFUs.

Recent studies have identified SDF-1 as a local factor regulating hematopoietic stem and progenitor cell function. SDF-1, binding to its cognate receptor CXCR4, regulates stem-cell homing and differentiation (19). This cytokine is also crucial for mobilization of stem cells from the bone marrow to the peripheral circulation and subsequent engraftment into diseased tissues (20). Our finding that SDF-1 showed significantly higher plasma levels during the proliferative phase and a negative correlation with EPC-CFUs suggests an important role for SDF-1 in homing of EPCs to the sites of neovascularization, in this case the endometrium.

In addition, our results support the novel role of SDF-1 during $\mathrm{MC}$ since it is negatively correlated to the percentage of $\mathrm{CD} 4^{+}$cells. This finding is supported by Sengenes et al (21), who found that SDF-1 induces the differentiation of $\mathrm{CD}_{3}{ }^{+}$cells toward an EC phenotype.

Furthermore, we investigated the local expression of SDF-1 in endometrial biopsies, and found that the endometrial level of SDF-1 was highest in the secretory phase, while circulating SDF-1 levels were highest during the proliferative phase. This indicates that the endometrial expression of SDF-1 may facilitate homing of circulating EPCs.

Recent studies indicate that the interplay between SDF-1, present in the endothelium, and EPC adhesion molecules is the main driving force behind the EPC recruitment process in vivo $(20,22)$. SDF-1 is believed to activate EPC integrins involved in the extravasation process. Yamaguchi et al (19) showed that local SDF-1 administration induces the accumulation of transplanted EPCs at ischemia sites and enhances neovascularization after EPC transplantation.

Previously, studies investigating the influence of the MC on the regulation of EPCs showed contradictory results. Robb et al, using quantification of EPCs by flow cytometry

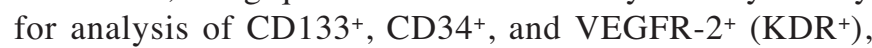
showed that the number of EPCs was higher in the proliferative phase compared with the periovulatory phase, while the number of EPCs using the EPC-CFU assay did not vary over the MC (5). By contrast, Matsubara et al (23) found that EPCs were increased in the secretory phase; this is in agreement with the present study, which showed a larger scatter of EPC-CFUs at ovulation, with a tendency towards increased numbers of CFUs during the secretory phase $(\mathrm{p}=0.40)$.

In this study, we did not perform quantification of EPCs by flow cytometry using CD $133^{+}, \mathrm{CD} 34^{+}$, and VEGFR-2 ${ }^{+}$. The
Table I. Principal cyclic variation of circulating and endometrial stromal cell-derived factor-1 (SDF-1) and circulating endothelial progenitor cell colony-forming units (EPC-CFUs).

\begin{tabular}{lcc}
\hline & \multicolumn{2}{c}{ Menstrual phases } \\
\cline { 2 - 3 } & Proliferative & Secretory \\
\hline Circulating SDF-1 & + & - \\
Endometrial SDF-1 & - & + \\
EPC-CFUs & - & + \\
\hline
\end{tabular}

in vitro function and clonogenic capacity of these EPCs have been evaluated elsewhere using CFU assays (10). However, it is not clear whether cells quantified by flow cytometry (i.e., by phenotype) and the cells forming the EPC-CFUs are the same populations, since the latter method may also select a subpopulation of myeloid cells (9). We reasoned that if EPCCFU represents the frequency/functionality of EPCs, then the formation of the EPC-CFU would be affected by the systemic variation of angiogenesis growth factors and EPC-mobilizing and homing factors.

In contrast to a previous study by Agrawal et al (24), but in agreement with Farha et al (25) and Gargett et al (26), the changes in VEGF in our study were not significantly different at the different time points studied. In fact, no clear cyclic pattern of VEGF or VEGFRs has been demonstrated during the MC $(27,28)$.

Granulocyte CSF and GM-CSF, which also release endothelial and myeloid precursors from the bone marrow (widely used to harvest myeloid cells for transplantation purposes), were not measurable here. This suggests, on the one hand, that there was no significant inflammatory reaction and, on the other hand, that these factors are not involved in EPC mobilization during the MC. This is in disagreement with the study of Cho et al (29) who, using another model with intravascular radiation therapy, found that GM-CSF treatment mobilizes EPCs and accelerates re-endothelialization.

To conclude, in this clinical study based on correlative data, our results suggest that during the proliferative MC phase, the endometrium prepares for recruiting new ECs to cover the new blood vessels (which may be generated by means of sprouting and intussusception). This fairly late recruitment may be accomplished by means of higher SDF-1 plasma levels. The origin of this SDF-1 is not known, but it may be speculated to originate from the endothelium. Subsequently, these EPCs are mobilized and home to the endometrium by means of higher tissue SDF-1 levels. However, we cannot exclude different scenarios including, for example, the possibility that low blood EPC levels during the proliferative phase are due to intensive trafficking of EPCs from the bone marrow, with a high uptake in the endometrium. Nonetheless, plasma and tissue levels of SDF-1 may be a novel functional marker of endometrial angiogenesis. Furthermore, using the MC as an angiogenic model, these issues can yield new insights into the mechanisms for the intense angiogenesis of the fertile endometrium. Moreover, 
our results provide an opportunity to identify conditions signified by abnormal angiogenesis, such as endometriosis, heavy uterine bleeding, and cancer. However, our results need to be further explored in a mechanistic experimental study.

\section{Acknowledgements}

The authors wish to thank Eva Wärdell of the Karolinska Institutet, Stockholm, Sweden, for software development. This study was supported by grants from the Swedish Medical Research Council (71X-05991, 71BI-14589), the Karolinska Institutet, Karolinska University Hospital Huddinge, the Cancer Society of Stockholm, and the Swedish Labor Market Insurance.

\section{References}

1. Salamonsen LA, Kovacs GT and Findlay JK: Current concepts of the mechanisms of menstruation. Baillieres Best Pract Res Clin Obstet Gynaecol 13: 161-179, 1999.

2. Gargett CE and Rogers PA: Human endometrial angiogenesis. Reproduction 121: 181-186, 2001.

3. Asahara T, Masuda H, Takahashi T, et al: Bone marrow origin of endothelial progenitor cells responsible for postnatal vasculogenesis in physiological and pathological neovascularization. Circ Res 85: 221-228, 1999

4. Mints M, Jansson M, Sadeghi B, et al: Endometrial endothelial cells are derived from donor stem cells in a bone marrow transplant recipient. Hum Reprod 23: 139-143, 2008.

5. Robb AO, Mills NL, Smith IB, et al: Influence of menstrual cycle on circulating endothelial progenitor cells. Hum Reprod 24: 619-625, 2009.

6. Asahara T, Murohara T, Sullivan A, et al: Isolation of putative progenitor endothelial cells for angiogenesis. Science 275: 964-967, 1997

7. Wassmann S, Werner N, Czech T and Nickenig G: Improvement of endothelial function by systemic transfusion of vascular progenitor cells. Circ Res 99: e74-e83, 2006.

8. Peichev M, Naiyer AJ, Pereira D, et al: Expression of VEGFR-2 and $\mathrm{AC} 133$ by circulating human $\mathrm{CD} 34(+)$ cells identifies a population of functional endothelial precursors. Blood 95: 952-958, 2000.

9. Prater DN, Case J, Ingram DA and Yoder MC: Working hypothesis to redefine endothelial progenitor cells. Leukemia 21: 1141-1149, 2007.

10. Hill JM, Zalos G, Halcox JP, et al: Circulating endothelial progenitor cells, vascular function, and cardiovascular risk. N Engl J Med 348: 593-600, 2003.

11. Blann AD, Woywodt A, Bertolini F, et al: Circulating endothelial cells. Biomarker of vascular disease. Thromb Haemost 93: 228-235, 2005.

12. Woywodt A, Bahlmann FH, De Groot K, Haller H and Haubitz M: Circulating endothelial cells: life, death, detachment and repair of the endothelial cell layer. Nephrol Dial Transplant 17: 1728-1730, 2002.

13. Capoccia BJ, Shepherd RM and Link DC: G-CSF and AMD3100 mobilize monocytes into the blood that stimulate angiogenesis in vivo through a paracrine mechanism. Blood 108: 2438-2445, 2006.
14. Minamino K, Adachi Y, Okigaki M, et al: Macrophage colonystimulating factor (M-CSF), as well as granulocyte colonystimulating factor (G-CSF), accelerates neovascularization. Stem Cells 23: 347-354, 2005.

15. Zemani F, Silvestre JS, Fauvel-Lafeve F, et al: Ex vivo priming of endothelial progenitor cells with SDF-1 before transplantation could increase their proangiogenic potential. Arterioscler Thromb Vasc Biol 28: 644-650, 2008.

16. Zheng H, Fu G, Dai T and Huang H: Migration of endothelial progenitor cells mediated by stromal cell-derived factor1alpha/CXCR4 via PI3K/Akt/eNOS signal transduction pathway. J Cardiovasc Pharmacol 50: 274-280, 2007.

17. Mints M, Blomgren B, Falconer C, Fianu-Jonasson A and Palmblad J: Microvascular density, vascular endothelial growth factor A, and its receptors in endometrial blood vessels in patients with menorrhagia. Fertil Steril 84: 692-700, 2005.

18. Mints M, Hultenby K, Zetterberg E, et al: Wall discontinuities and increased expression of vascular endothelial growth factor-A and vascular endothelial growth factor receptors 1 and 2 in endometrial blood vessels of women with menorrhagia. Fertil Steril 88: 691-697, 2007.

19. Yamaguchi J, Kusano KF, Masuo O, et al: Stromal cell-derived factor-1 effects on ex vivo expanded endothelial progenitor cell recruitment for ischemic neovascularization. Circulation 107: 1322-1328, 2003.

20. Lapidot T, Dar A and Kollet O: How do stem cells find their way home? Blood 106: 1901-1910, 2005.

21. Sengenes C, Miranville A, Maumus M, de Barros S, Busse R and Bouloumie A: Chemotaxis and differentiation of human adipose tissue $\mathrm{CD} 34^{+} / \mathrm{CD} 31^{-}$progenitor cells: role of stromal derived factor- 1 released by adipose tissue capillary endothelial cells. Stem Cells 25: 2269-2276, 2007.

22. Jin DK, Shido K, Kopp HG, et al: Cytokine-mediated deployment of SDF-1 induces revascularization through recruitment of CXCR4+ hemangiocytes. Nat Med 12: 557-567, 2006.

23. Matsubara K, Abe E, Matsubara Y, Kameda K and Ito M: Circulating endothelial progenitor cells during normal pregnancy and pre-eclampsia. Am J Reprod Immunol 56: 79-85, 2006.

24. Agrawal R, Conway GS, Sladkevicius P, et al: Serum vascular endothelial growth factor (VEGF) in the normal menstrual cycle: association with changes in ovarian and uterine Doppler blood flow. Clin Endocrinol (Oxf) 50: 101-106, 1999.

25. Farha S, Asosingh K, Laskowski D, et al: Pulmonary gas transfer related to markers of angiogenesis during the menstrual cycle. J Appl Physiol 103: 1789-1795, 2007.

26. Gargett CE, Lederman FL, Lau TM, Taylor NH and Rogers PA: Lack of correlation between vascular endothelial growth factor production and endothelial cell proliferation in the human endometrium. Hum Reprod 14: 2080-2088, 1999.

27. Shifren JL, Tseng JF, Zaloudek CJ, et al: Ovarian steroid regulation of vascular endothelial growth factor in the human endometrium: implications for angiogenesis during the menstrual cycle and in the pathogenesis of endometriosis. J Clin Endocrinol Metab 81: 3112-3118, 1996.

28. Li XF, Gregory J and Ahmed A: Immunolocalisation of vascular endothelial growth factor in human endometrium. Growth Factors 11: 277-282, 1994.

29. Cho HJ, Kim HS, Lee MM, et al: Mobilized endothelial progenitor cells by granulocyte-macrophage colony-stimulating factor accelerate reendothelialization and reduce vascular inflammation after intravascular radiation. Circulation 108: 2918-2925, 2003. 